\title{
LV-OP-3-1
}

\section{Surgical outcomes of left side hepatectomy compared with right side hepatectomy in type I, II or IV hilar cholangiocellular carcinoma}

\author{
Seung Soo HONG, Dai Hoon HAN, Kyung Sik KIM, Jin Sub CHOI, Gi Hong CHOI*
}

Division of Hepatopancreaticobiliary Surgery, Department of Surgery, Yonsei University College of Medicine, Seoul, Korea

Introduction: Right-side hepatectomy (RH) has been considered an oncological resection for hilar cholangiocarcinoma (HCCC), however the decision for left-side hepatectomy (LH) or RH is still a controversial issue. We compared surgical and oncologic outcomes of LH and RH in HCCC type I, II or IV in which either side of hepatectomy was expected to have a negative margin.

Methods: From 2001 to 2020, 85 patients underwent major liver resection for type I, II or IV HCCC. Among them, 52 patients who had no vascular invasion, no unilateral tumor growth and no atrophy of unilateral liver were enrolled. Preoperative characteristics, perioperative outcomes and long-term outcomes were compared between RH $(n=29)$ and LH $(n=23)$ group.

Results: Clinical characteristics and disease severity did not differ between two groups. Portal vein embolization rate (RH: $48.3 \%$ vs. LH: $0.0 \%, p<0.001)$ and duration of diagnosis to operation (RH: $31.0 \pm 16.2$ vs. LH: $18.8 \pm 13.4, p=0.006)$ were significantly higher in RH group. Postoperative hepatic failure (over grade B) was more frequent in RH group (RH: 55.2\% vs LH: $21.7 \%, p=0.015$ ). R0 resection rate (RH: $72.4 \%$ vs. LH: $78.3 \%, p=0.629)$, median disease free $(p=0.620)$ and overall survival $(p=0.487)$ did not differ between two groups. R1 resection and number of lymph node metastasis were significant risk factor for DFS in multivariate analysis.

Conclusions: In type I, II or type IV HCCC in which either LH or RH was a feasible option, LH provided shorter period of preoperative preparation, lower postoperative hepatic failure rate, similar R0 rate and comparable long-term outcomes compared with RH. Therefore, LH should be considered reasonable option to type I, II or IV HCCC. 